\title{
Discrete Symmetries and Generalized Fields of Dyons
}

\author{
P. S. Bisht ${ }^{(1,2)}$, Tianjun Li ${ }^{(2)}$, Pushpa ${ }^{(1)}$ and O. P. S. Negi ${ }^{(1)}$
}

November 2, 2018

1. Department of Physics, Kumaun University,

S. S. J. Campus, Almora- 263601(Uttarakhand), India

2. Institute of Theoretical Physics, Chinese Academy of Sciences,

P. O. Box 2735, Beijing 100080, P. R. China
Email: ps_bisht123@rediffmail.com, tli@itp.ac.cn, pushpakalauni60@yahoo.co.in, ops_negi@yahoo.co.in

\begin{abstract}
We have studied the different symmetric properties of the generalized Maxwell's - Dirac equation along with their quantum properties. Applying the parity $(\mathcal{P})$, time reversal $(\mathcal{T})$, charge conjugation $(\mathcal{C})$ and their combined effect like parity time reversal $(\mathcal{P} \mathcal{T})$, charge conjugation and parity $(\mathcal{C P})$ and $\mathcal{C P} T$ transformations to varius equations of generalized fields of dyons, it is shown that the corresponding dynamical quantities and equations of dyons are invariant under these discrete symmetries.
\end{abstract}

Key words- parity, time reversal, charge-conjugation, dyons

PACS No.- 14.80 Hv. 


\section{Introduction}

In developing the standard model for particles, certain types of interactions and decays are observed to be common and others seem to be forbidden. The study of interactions has led to a number of conservation laws which govern them [1, 2, 3, 4,. These conservation laws are in addition to the classical conservation laws such as conservation of energy, charge, etc., which still apply in the realm of particle interactions. Specific quantum numbers have been assigned to the different fundamental particles, and other conservation laws are associated with those quantum numbers. From another point of view, it would seem that any localized particle of finite mass should be unstable, since the decay into several smaller particles provides many more ways to distribute the energy, and thus would have higher entropy. This idea is even stated as a principle called the "totalitarian principle" which might be stated as "every process that is not forbidden must occur". From this point of view, any decay process which is expected but not observed must be prevented from occurring by some conservation law. This approach has been fruitful in helping to determine the rules for particle decay. Conservation laws for parity, isospin, and strangeness have been developed by detailed observation of particle interactions [1, 2, 3, 4]. The implications of parity symmetry in atoms were first investigated by Wigner [5], who demonstrated that the combination of charge conjugation $(\mathcal{C})$, parity $(\mathcal{P})$ and time reversal $(\mathcal{T})$ is considered to be a fundamental symmetry operation - all physical particles and interactions appear to be invariant under this combination. Called $\mathcal{C P} \mathcal{T}$ invariance, this symmetry plumbs the depths of our understanding of nature. Many of the researchers already described the behavior of these symmetries in electromagnetic fields [6, 7, 8, 9, 10, 11, 12, 13, 14, 15, 16, 17. In a series of papers [18, 19, 20, 21, 22, we have discussed the generalized Dirac Maxwell (GDM) equations in presence of electric and magnetic sources in an isotropic (homogeneous) medium. We have also analyzed the other quantum equations of dyons in consistent and manifest covariant way [18]. This theory has been shown to remain invariant under the duality transformations in isotropic homogeneous medium. Quaternion analysis of time dependent Maxwell's equations has been developed [19] in presence of electric and magnetic charges and the solution for the classical problem of moving charge (electric and magnetic) are obtained consistently. The time dependent generalized Dirac - Maxwell's (GDM) equations of dyons have also been discussed [20] in chiral and inhomogeneous media and the solutions for the classical problem are obtained. The quaternion reformulation of generalized electromagnetic fields of dyons in chiral and inhomogeneous media has also been analyzed [21]. We have also discussed [22] the monochromatic fields of generalized electromagnetic fields of dyons in slowly changing media in a consistent manner. In this paper, we have made an attempt to study the different symmetric properties of generalized Maxwell's Dirac equations in the homogeneous medium. It is shown that the generalized Maxwell's - Dirac (GDM) equations are invariant under discrete symmetries like parity $\mathcal{P}$, time reversal $\mathcal{T}$, charge conjugation $(\mathcal{C})$, parity - time reversal operation $\mathcal{P} \mathcal{T}$, charge conjugation - parity operation $\mathcal{C P}$ and combined operation $(\mathcal{C P} \mathcal{T})$. But when we take the compact form of these equations as complex ones for generalized fields of dyons, we observe that field equation and equation of motion of dyons are no more invariant under $\mathcal{P}$ symmetry, $\mathcal{T}$ transformations and combined effects of $\mathcal{C P}$ and $\mathcal{P} \mathcal{T}$. On the other hand, these equa- 
tions and other quantum equations of dyons in homogeneous medium are invariant under the combined operation $(\mathcal{C P} \mathcal{T})$. As such, we may conclude that $\mathcal{C P} \mathcal{T}$ invariance is an exact symmetry for generalized fields of dyons and reproduces the consistent theory of electric charge (magnetic monopole) in the absence magnetic monopole (electric charge ) on dyons.

\section{Fields Associated with dyons}

Assuming the existence of magnetic monopoles, Dirac [23] generalized the Maxwell's equations in homogeneous isotropic medium written in the following manner in SI units $(c=\hbar=1)$ i. e.

$$
\begin{aligned}
& \vec{\nabla} \cdot \vec{E}= \frac{\rho_{e}}{\varepsilon} ; \\
& \vec{\nabla} \cdot \vec{B}=\mu \rho_{m} ; \\
& \vec{\nabla} \times \vec{E}=-\frac{\partial \vec{B}}{\partial t}-\frac{\overrightarrow{j_{m}}}{\varepsilon} ; \\
& \vec{\nabla} \times \vec{B}=\frac{1}{v^{2}} \frac{\partial \vec{E}}{\partial t} \quad+\mu \quad \overrightarrow{j_{e}} ;
\end{aligned}
$$

where $\rho_{e}$ and $\rho_{m}$ are respectively the electric and magnetic charge densities while $\overrightarrow{j_{e}}$ and $\overrightarrow{j_{m}}$ are the corresponding current densities,$\varepsilon$ and $\mu$ are defined respectively as relative permittivity and permeability in electric and magnetic fields in the medium, $\vec{E}$ is electric field, $\vec{B}$ is magnetic field and $v=\frac{1}{\sqrt{\mu \varepsilon}}$ is the velocity of the electromagnetic wave. Differential equation (11) are the generalized field equation of dyon in homogeneous medium and the corresponding electric and magnetic fields are called the generalized electromagnetic fields of dyons. These electromagnetic fields of dyons are expressed in following differential form in homogeneous medium in terms of two four potentials as [19],

$$
\begin{aligned}
& \vec{E}=-\vec{\nabla} \phi_{e}-\frac{\partial \vec{C}}{\partial t}-\vec{\nabla} \times \vec{D} \\
& \vec{B}=-\vec{\nabla} \phi_{m}-\frac{1}{v^{2}} \frac{\partial \vec{D}}{\partial t}+\vec{\nabla} \times \vec{C}
\end{aligned}
$$

where $\left\{C^{\mu}\right\}=\left\{\phi_{e}, v \vec{C}\right\}$ and $\left\{D^{\mu}\right\}=\left\{v \phi_{m}, \vec{D}\right\}$ are the two four - potentials respectively associated with electric and magnetic charges. Let us define the complex vector field $\vec{\psi}$ in the following form,

$$
\vec{\psi}=\vec{E}-i v \vec{B}
$$

Equations (11,21) and (3), thus give rise to the following relation between generalized field and the components of the generalized four - potential as; 


$$
\vec{\psi}=-\frac{\partial \vec{V}}{\partial t}-\vec{\nabla} \phi-i v(\vec{\nabla} \times \vec{V}) .
$$

Here $\left\{V_{\mu}\right\}$ is the generalized four - potential of dyons in homogeneous medium and defined as ;

$$
\left\{V_{\mu}\right\}=\{\phi,-\vec{V}\}
$$

where

$$
\phi=\phi_{e}-\quad i \quad v \phi_{m}
$$

and

$$
\vec{V}=\vec{C}-i \frac{\vec{D}}{v}
$$

Using equations (3, 4,5,6), Dirac-Maxwell field equation ( 1 ) may be written in terms of generalized field $\vec{\psi}$ as;

$$
\begin{array}{ccc}
\vec{\nabla} \cdot \vec{\psi} & = & \frac{\rho}{\varepsilon} ; \\
\vec{\nabla} \times \vec{\psi}=-i v\left(\mu \vec{J}+\frac{1}{v^{2}}\right. & \left.\frac{\partial \vec{\psi}}{\partial t}\right) ;
\end{array}
$$

where $\rho$ and $\vec{J}$ are respectively the generalized charge and current source densities of dyons in homogeneous medium [19] given by;

$$
\begin{aligned}
& \rho=\rho_{e} \quad-i \quad \frac{\rho_{m}}{v} ; \\
& J=j_{e}-i v \quad j_{m} .
\end{aligned}
$$

With the use of equations (18) and (99), we introduce a new parameter $\vec{S}$ (i.e. the field current) as

$$
\vec{S}=\square \vec{\psi}=-\mu \frac{\partial \vec{J}}{\partial t}-\frac{1}{\varepsilon} \vec{\nabla} \rho-i v \mu(\vec{\nabla} \times \vec{J}) ;
$$

where $\square$ is the D'Alembertian operator expressed as

$$
\square \vec{\psi}==\frac{1}{v^{2}} \frac{\partial^{2}}{\partial t^{2}}-\nabla^{2}=\frac{1}{v^{2}} \frac{\partial^{2}}{\partial t^{2}}-\frac{\partial^{2}}{\partial x^{2}}-\frac{\partial^{2}}{\partial y^{2}}-\frac{\partial^{2}}{\partial z^{2}}
$$


where $v$ is the speed of electromagnetic wave in homogeneous isotropic medium. In terms of complex potential, the field equation (11) is written as

$$
\begin{aligned}
\square \phi & =v \mu \rho \\
\square \vec{V} & =\mu \vec{J}
\end{aligned}
$$

Hence, we may write the tensorial form of generalized Maxwell Dirac equation (1) of dyons in isotropic homogeneous medium as,

$$
\begin{aligned}
& F_{\mu \nu, \nu}=j_{\mu}^{e} ; \\
& F_{\mu \nu, \nu}^{d}=j_{\mu}^{m} ;
\end{aligned}
$$

where

$$
\begin{aligned}
\left\{j_{\mu}^{e}\right\} & =\left\{v \rho_{e}, \overrightarrow{j_{e}}\right\} \\
\left\{j_{\mu}^{m}\right\} & =\left\{\rho_{m}, \vec{v} \overrightarrow{j_{m}}\right\}
\end{aligned}
$$

Defining the generalized field tensor of dyons [19] as;

$$
G_{\mu \nu, \nu}=F_{\mu \nu}-i \quad v F_{\mu \nu}^{d}
$$

one can directly obtain the following form of generalized field equation of dyon in homogeneous isotropic medium as

$$
G_{\mu \nu, \nu}=J_{\mu}
$$

where

$$
\left\{J_{\mu}\right\}=\{\rho,-\vec{J}\} .
$$

The Lorentz four - force equation of motion for dyon in homogeneous isotropic medium is written as;

$$
f_{\mu}=m_{0} \ddot{x}_{\mu}=\operatorname{Re} Q^{*}\left(G_{\mu \nu} u^{\nu}\right) ;
$$

where ' $R e^{\prime}$ denotes the real part, $\left\{\ddot{x}_{\mu}\right\}$ is the four - acceleration and $\left\{u^{\nu}\right\}$ is the four velocity of the particle and $Q$ is the generalized charge of dyon in homogeneous isotropic medium . 


\section{Effect of Parity on Generalized fields of dyons}

Parity is the symmetry of interaction which involves a transformation that changes the algebraic sign of the coordinate system [1]. Under the parity transformation $\mathcal{P}$;

$$
\mathcal{P}:\left(\begin{array}{l}
x \\
y \\
z
\end{array}\right) \mapsto\left(\begin{array}{l}
-x \\
-y \\
-z
\end{array}\right) .
$$

The parity transformation changes a right - handed coordinate system into a left handed one or vice versa. Under the parity transformations the physical quantities like time $(t)$, mass $(m)$, electric charge density $\left(\rho_{e}\right)$, magnetic current density $\left(\overrightarrow{j_{m}}\right)$, electric scalar potential $\left(\phi_{e}\right)$, magnetic vector potential $(\vec{D})$, generalized scalar potential $(\phi)$, time derivative $\left(\partial_{t}\right)$, D' Alembertian $(\square)$ and magnetic field $(\vec{B})$, have even parity as they do not change [16] their sign under spatial inversion (Parity transformation) showing that they have even parity.

$$
\begin{aligned}
\mathcal{P}(t) \mathcal{P}^{-1} & =t ; \\
\mathcal{P}(m) \mathcal{P}^{-1} & =m ; \\
\mathcal{P}\left(\rho_{e}\right) \mathcal{P}^{-1} & =\rho_{e} ; \\
\mathcal{P}\left(\overrightarrow{j_{m}}\right) \mathcal{P}^{-1} & =\overrightarrow{j_{m}} \\
\mathcal{P}\left(\phi_{e}\right) \mathcal{P}^{-1} & =\phi_{e} ; \\
\mathcal{P}(\vec{D}) \mathcal{P}^{-1} & =\vec{D} ; \\
\mathcal{P}\left(\partial_{t}\right) \mathcal{P}^{-1} & =\partial_{t} ; \\
\mathcal{P}(\square) \mathcal{P}^{-1} & =\square ; \\
\mathcal{P}(\vec{B}) \mathcal{P}^{-1} & =\vec{B} .
\end{aligned}
$$

On the other hand, the physical like displacement $(x)$, velocity $(\vec{u})$, acceleration $(\vec{a})$, magnetic charge density $\left(\rho_{m}\right)$, electric current density $\left(\overrightarrow{j_{e}}\right)$, magnetic scalar potential $\left(\phi_{m}\right)$, differential operator $(\vec{\nabla})$, electric vector potential $(\vec{C})$ and electric field $(\vec{E})$ have odd parity as they change [16] their sign under spatial inversion (Parity transformation) i.e.

$$
\begin{aligned}
\mathcal{P}(x) \mathcal{P}^{-1} & =-x ; \\
\mathcal{P}(\vec{u}) \mathcal{P}^{-1} & =-\vec{u} ; \\
\mathcal{P}(\vec{a}) \mathcal{P}^{-1} & =-\vec{a} ; \\
\mathcal{P}\left(\rho_{m}\right) \mathcal{P}^{-1} & =\rho_{m} ; \\
\mathcal{P}\left(\overrightarrow{j_{e}}\right) \mathcal{P}^{-1} & =-\overrightarrow{j_{e}} ; \\
\mathcal{P}(\phi) \mathcal{P}^{-1} & =-\phi_{m} \\
\mathcal{P}(\vec{\nabla}) \mathcal{P}^{-1} & =-\vec{\nabla} \\
\mathcal{P}(\vec{C}) \mathcal{P}^{-1} & =-\vec{C} \\
\mathcal{P}(\vec{E}) \mathcal{P}^{-1} & =-\vec{E}
\end{aligned}
$$


showing that due to change of sign, these quantity have odd parity. Now applying the parity transformations to equation (1), we find that the generalized Dirac - Maxwell's equations (1) are invariant under parity transformations. On applying the parity transformation to equations (4, 6, 7, 9, 10), we get

$$
\begin{aligned}
\mathcal{P}(\vec{\psi}) \mathcal{P}^{-1} & =-\vec{\psi} ; \\
\mathcal{P}(\phi) \mathcal{P}^{-1} & =\phi ; \\
\mathcal{P}(\vec{V}) \mathcal{P}^{-1} & =-\vec{V} ; \\
\mathcal{P}(\rho) \mathcal{P}^{-1} & =\rho ; \\
\mathcal{P}(\vec{J}) \mathcal{P}^{-1} & =-\vec{J} ; \\
\mathcal{P}(\vec{S}) \mathcal{P}^{-1} & =-\vec{S} .
\end{aligned}
$$

As such, the components of generalized complex potential given by equations (12) are invariant under parity transformations. The tensorial form of generalized Maxwell Dirac equation of dyons in isotropic homogeneous medium given by equations (13) are also invariant under parity transformations. On the other hand, the generalized field equations (16) and the Lorentz four - force equation of motion (18) for generalized fields of dyon in homogeneous isotropic medium loose their invariance under parity transformations.

\section{Effect of Time reversal on Generalized fields of dyons}

In simple classical terms, time reversal just means replacing $t$ by $-t$, inverting the direction of the flow of time [2]. Reversing time also reverses the time derivatives of spatial quantities. So, it reverses momentum and angular momentum. Thus the following physical quantities like displacement $(x)$, Acceleration $(a)$, electric charge source density $\left(\rho_{e}\right)$, magnetic current source density $\left(\overrightarrow{j_{m}}\right)$, electric scalar potential $\left(\phi_{e}\right)$, magnetic vector potential $(\vec{D})$, space derivative $(\vec{\nabla})$ and generalized electric field $(\vec{E})$ are unaffected and do not change [16] their sign under time reversal i.e.

$$
\begin{aligned}
\mathcal{T}(x) \mathcal{T}^{-1} & =x ; \\
\mathcal{T}(\vec{a}) \mathcal{T}^{-1} & =\vec{a} ; \\
\mathcal{T}\left(\rho_{e}\right) \mathcal{T}^{-1} & =\rho_{e} ; \\
\mathcal{T}\left(\overrightarrow{j_{m}}\right) \mathcal{T}^{-1} & =\overrightarrow{j_{m}} ; \\
\mathcal{T}\left(\phi_{e}\right) \mathcal{T}^{-1} & =\phi_{e} ; \\
\mathcal{T}(\vec{D}) \mathcal{T}^{-1} & =\vec{D} ; \\
\mathcal{T}(\vec{\nabla}) \mathcal{T}^{-1} & =\vec{\nabla} ; \\
\mathcal{T}(\vec{E}) \mathcal{T}^{-1} & =\vec{E} .
\end{aligned}
$$

On the other hand the physical quantities like time $(t)$, particle velocity $(\vec{u})$,electric current source density $\left(\overrightarrow{j_{e}}\right)$, magnetic scalar potential $\left(\phi_{m}\right)$, electric vector potential $(\vec{C})$, time derivative $\left(\partial_{t}\right)$ and generalized magnetic field $(\vec{B})$ are affected and change their sign under time reversal i.e.. 


$$
\begin{aligned}
\mathcal{T}(t) \mathcal{T}^{-1} & =-t ; \\
\mathcal{T}(\vec{u}) \mathcal{T}^{-1} & =-\vec{u} ; \\
\mathcal{T}\left(\overrightarrow{j_{e}}\right) \mathcal{T}^{-1} & =-\overrightarrow{j_{e}} \\
\mathcal{T}\left(\phi_{m}\right) \mathcal{T}^{-1} & =-\phi_{m} \\
\mathcal{T}(\vec{C}) \mathcal{T}^{-1} & =-\vec{C} \\
\mathcal{T}\left(\partial_{t}\right) \mathcal{T}^{-1} & =-\partial_{t} \\
\mathcal{T}(\vec{B}) \mathcal{T}^{-1} & =-\vec{B}
\end{aligned}
$$

Applying the time reversal $(\mathcal{T})$ symmetry to equation (1), we find that the generalized Dirac - Maxwell equations (1) in the homogeneous medium are invariant under $\mathcal{T}$ symmetry. Similarly, on applying $\mathcal{T}$ symmetry to equations (4,6,7, 8,10), we get

$$
\begin{aligned}
& \mathcal{T}(\vec{\psi}) \mathcal{T}^{-1}=\vec{\psi} ; \\
& \mathcal{T}(\phi) \mathcal{T}^{-1}=\phi ; \\
& \mathcal{T}(\vec{V}) \mathcal{T}^{-1}=-\vec{V} ; \\
& \mathcal{T}(\rho) \mathcal{T}^{-1}=\rho ; \\
& \mathcal{T}(\vec{J}) \mathcal{T}^{-1}=-\vec{J} ; \\
& \mathcal{T}(\vec{S}) \mathcal{T}^{-1}=\vec{S}
\end{aligned}
$$

Thus, the components of generalized complex potential defined by equation (12) are invariant under $\mathcal{T}$ symmetry. As such, the tensorial forms of generalized Dirac- Maxwell equation of dyons in isotropic homogeneous medium given by equation (13) remain invariant under the time reversal $(\mathcal{T})$ symmetry while the generalized equations (16) and (18) describe respectively the field equation and Lorentz force equation of motion for dyons are not invariant under $\mathcal{T}$ symmetry like parity.

\section{Dyon fields under Charge Conjugation}

Classically, charge conjugation (Charge symmetry) replaces positive charges by negative charges and vice versa [3]. Since electric and magnetic fields have their origins in charges, one can reverse these fields on the application of charge conjugation. Charge conjugation also involves reversing all the internal quantum numbers like lepton number, baryon number and strangeness etc. The physical quantities like displacement $(x)$, time $(t)$, mass $(m)$, particle velocity $(u)$, acceleration $(a)$, space derivative $(\vec{\nabla})$ and time derivative $\left(\partial_{t}\right)$ are invariant under charge conjugation [16] while the energy, momentum or spin are unaffected i.e. 


$$
\begin{aligned}
& \mathcal{C}(x) \mathcal{C}^{-1}=x ; \\
& \mathcal{C}(t) \mathcal{C}^{-1}=t \\
& \mathcal{C}(m) \quad \mathcal{C}^{-1}=m ; \\
& \mathcal{C}(\vec{u}) \mathcal{C}^{-1}=\vec{u} ; \\
& \mathcal{C}(\vec{a}) \mathcal{C}^{-1}=\vec{a} \\
& \mathcal{C}(\vec{\nabla}) \mathcal{C}^{-1}=\vec{\nabla} ; \\
& \mathcal{C}\left(\partial_{t}\right) \mathcal{C}^{-1}=\partial_{t} \text {. }
\end{aligned}
$$

On the other hand the physical quantities like electric charge $(e)$, magnetic charge $(g)$, electric charge source density $\left(\rho_{e}\right)$, magnetic charge source density $\left(\rho_{m}\right)$, generalized charge source density $(\rho)$, electric scalar potential $\left(\phi_{e}\right)$, magnetic scalar potential $\left(\phi_{m}\right)$, generalized scalar potential $(\phi)$,electric current source density $\left(\overrightarrow{j_{e}}\right)$, magnetic current source density $\left(\overrightarrow{j_{m}}\right)$, generalized current source density $(\vec{j})$, electric vector potential $(\vec{C})$, magnetic vector potential $(\vec{D})$, generalized vector potential $(\vec{V})$, generalized electric field $(\vec{E})$ and generalized magnetic field $(\vec{B})$ are directly related to the charge and thus their sign is changed under charge symmetry as,

$$
\begin{aligned}
\mathcal{C}(e) \mathcal{C}^{-1} & =-e ; \\
\mathcal{C}(g) \mathcal{C}^{-1} & =-g ; \\
\mathcal{C}\left(\rho_{e}\right) \mathcal{C}^{-1} & =-\rho_{e} \\
\mathcal{C}\left(\rho_{m}\right) \mathcal{C}^{-1} & =-\rho_{m} \\
\mathcal{C}(\rho) \mathcal{C}^{-1} & =-\rho ; \\
\mathcal{C}\left(\phi_{e}\right) \mathcal{C}^{-1} & =-\phi_{e} \\
\mathcal{C}\left(\phi_{m}\right) \mathcal{C}^{-1} & =-\phi_{m} \\
\mathcal{C}(\phi) \mathcal{C}^{-1} & =-\phi ; \\
\mathcal{C}\left(\overrightarrow{j_{e}}\right) \mathcal{C}^{-1} & =-\overrightarrow{j_{e}} \\
\mathcal{C}\left(\overrightarrow{j_{m}}\right) \mathcal{C}^{-1} & =-\overrightarrow{j_{m}} \\
\mathcal{C}(\vec{J}) \mathcal{C}^{-1} & =-\vec{J} \\
\mathcal{C}(\vec{C}) \mathcal{C}^{-1} & =-\vec{C} \\
\mathcal{C}(\vec{D}) \mathcal{C}^{-1} & =-\vec{D} \\
\mathcal{C}(\vec{V}) \mathcal{C}^{-1} & =-\vec{V} \\
\mathcal{C}(\vec{E}) \mathcal{C}^{-1} & =-\vec{E} \\
\mathcal{C}(\vec{B}) \mathcal{C}^{-1} & =-\vec{B}
\end{aligned}
$$

If we check the $\mathcal{C}$ invariance of equation (1), we find that the generalized Dirac Maxwell's equations (1) of dyons in the homogeneous medium are invariant under charge symmetry. Similarly if we apply the $\mathcal{C}$ invariance conditions to equations (4) and (10), we see that the physical quantities like generalized field $(\vec{\psi})$ and generalized field current $(\vec{S})$ of dyon in homogeneous medium change their sign as, 


$$
\begin{aligned}
& \mathcal{C}(\vec{\psi}) \mathcal{C}^{-1}=-\vec{\psi} \\
& \mathcal{C}(\vec{S}) \mathcal{C}^{-1}=-\vec{S}
\end{aligned}
$$

Applying the $\mathcal{C}$ transformation to, the complex potential equations (12), the tensorial form of Dirac - Maxwell equations (13), generalized field equation (16) and Lorentz force equation (18) of dyons in isotropic homogeneous medium, we see that these equations of dyons in homogeneous isotropic medium are invariant under $\mathcal{C}$ transformations.

\section{Combined operation of Parity and Time Reversal $(\mathcal{P} \mathcal{T})$ and dyon fields}

The most widely known symmetry are based on group theory is called $\mathcal{P} \mathcal{T}$ symmetry. In case of $\mathcal{P} \mathcal{T}$ symmetry, the particle velocity, potential and current are invariant under the simultaneous action of the space and time reflection operators $\mathcal{P}$ and $\mathcal{T}$ [6] i.e.

$$
\begin{aligned}
(\mathcal{P} \mathcal{T})(\vec{u})(\mathcal{P} \mathcal{T})^{-1} & =\vec{u} \\
(\mathcal{P} \mathcal{T})\left(\rho_{e}\right)(\mathcal{P} \mathcal{T})^{-1} & =\rho_{e} \\
(\mathcal{P} \mathcal{T})\left(\rho_{m}\right)(\mathcal{P} \mathcal{T})^{-1} & =\rho_{m} \\
(\mathcal{P} \mathcal{T})(\rho)(\mathcal{P} \mathcal{T})^{-1} & =\rho \\
(\mathcal{P} \mathcal{T})\left(\overrightarrow{j_{e}}\right)(\mathcal{P} \mathcal{T})^{-1} & =\overrightarrow{j_{e}} \\
(\mathcal{P} \mathcal{T})\left(\overrightarrow{j_{m}}\right)(\mathcal{P} \mathcal{T})^{-1} & =\overrightarrow{j_{m}} \\
(\mathcal{P} \mathcal{T})(\vec{J})(\mathcal{P} \mathcal{T})^{-1} & =\vec{J} \\
(\mathcal{P} \mathcal{T})\left(\phi_{e}\right)(\mathcal{P} \mathcal{T})^{-1} & =\phi_{e} \\
(\mathcal{P} \mathcal{T})\left(\phi_{m}\right)(\mathcal{P} \mathcal{T})^{-1} & =\phi_{m} \\
(\mathcal{P} \mathcal{T})(\phi)(\mathcal{P} \mathcal{T})^{-1} & =\phi ; \\
(\mathcal{P} \mathcal{T})(\vec{C})(\mathcal{P} \mathcal{T})^{-1} & =\vec{C} \\
(\mathcal{P} \mathcal{T})(\vec{D})(\mathcal{P} \mathcal{T})^{-1} & =\vec{D} \\
(\mathcal{P} \mathcal{T})(\vec{V})(\mathcal{P} \mathcal{T})^{-1} & =\vec{V}
\end{aligned}
$$

where the symbols of physical quantities have their usual meaning as discussed above. On the other hand, the following physical variables with their usual symbols

$$
\begin{aligned}
(\mathcal{P} \mathcal{T})(x)(\mathcal{P} \mathcal{T})^{-1} & =-x ; \\
(\mathcal{P} \mathcal{T})(t)(\mathcal{P} \mathcal{T})^{-1} & =-t ; \\
(\mathcal{P} \mathcal{T})(\vec{a})(\mathcal{P} \mathcal{T})^{-1} & =-\vec{a} \\
(\mathcal{P} \mathcal{T})(\vec{\nabla})(\mathcal{P} \mathcal{T})^{-1} & =-\vec{\nabla} \\
(\mathcal{P} \mathcal{T})(\vec{E})(\mathcal{P} \mathcal{T})^{-1} & =-\vec{E} \\
(\mathcal{P} \mathcal{T})(\vec{B})(\mathcal{P} \mathcal{T})^{-1} & =-\vec{B}
\end{aligned}
$$


get changed their sign under $\mathcal{P} \mathcal{T}$ symmetry. Simultaneously, if we perform the $\mathcal{P} \mathcal{T}$ symmetry operation to the equation (11), we find that the generalized Dirac-Maxwell's equations of dyon in the homogeneous medium are invariant under $\mathcal{P} \mathcal{T}$ symmetry. Applying the $\mathcal{P} \mathcal{T}$ symmetry to generalized field $(\vec{\psi})$ and generalized field current $(\vec{S})$ of dyons in homogeneous medium respectively given by equations (44) and (10), we see that these quantities change their sign under $\mathcal{P} \mathcal{T}$ symmetry i.e.

$$
\begin{aligned}
& (\mathcal{P} \mathcal{T})(\vec{\psi})(\mathcal{P} \mathcal{T})^{-1}=-\vec{\psi} \\
& (\mathcal{P} \mathcal{T})(\vec{S})(\mathcal{P} \mathcal{T})^{-1}=-\vec{S}
\end{aligned}
$$

while the dyon field equations (11, 8, and 12) are invariant under $\mathcal{P} \mathcal{T}$ symmetry. The combined operation of $\mathcal{P} \mathcal{T}$ transformation also shows the invariance of the tensorial forms of Maxwell's equations (13) and the generalized field equation (16) of dyons in homogeneous isotropic medium. On the other hand, the Lorentz four - force equation (18) of motion for dyon in homogeneous isotropic medium looses its invariance under $\mathcal{P} \mathcal{T}$ symmetry and thus gets changed its sign under this symmetry.

\section{Charge Conjugation and Parity for field associated with dyons}

Associated with the conservation laws which govern the behavior of physical particles, charge conjugation $(\mathcal{C})$ and parity $(\mathcal{P})$ combine to constitute a fundamental symmetry called $\mathcal{C P}$ invariance [3, 4, 24]. The following quantities are invariant under the combined effect of $\mathcal{C P}$ i.e.

$$
\begin{aligned}
(\mathcal{C P})(t)(\mathcal{C P})^{-1} & =-t ; \\
(\mathcal{C P})(m)(\mathcal{C P})^{-1} & =m ; \\
(\mathcal{C P})\left(\partial_{t}\right)(\mathcal{C P})^{-1} & =\partial_{t} ; \\
(\mathcal{C P})\left(\rho_{m}\right)(\mathcal{C P})^{-1} & =\rho_{m} ; \\
(\mathcal{C P})\left(\overrightarrow{j_{e}}\right)(\mathcal{C P})^{-1} & =\overrightarrow{j_{e}} ; \\
(\mathcal{C P})\left(\phi_{m}\right)(\mathcal{C P})^{-1} & =\phi_{m} ; \\
(\mathcal{C P})(\vec{C})(\mathcal{C P})^{-1} & =\vec{C} ; \\
(\mathcal{C P})(\vec{E})(\mathcal{C P})^{-1} & =\vec{E}
\end{aligned}
$$

On the other hand, we see that the following parameters change their sign under the combined operation $\mathcal{C P}$ of charge conjugation and parity i.e. 


$$
\begin{aligned}
(\mathcal{C P})(x)(\mathcal{C P})^{-1} & =-x ; \\
(\mathcal{C P})(\vec{u})(\mathcal{C P})^{-1} & =-\vec{u} ; \\
(\mathcal{C P})(e)(\mathcal{C P})^{-1} & =-e ; \\
(\mathcal{C P})(g)(\mathcal{C P})^{-1} & =-g ; \\
(\mathcal{C P})(\vec{\nabla})(\mathcal{C P})^{-1} & =-\vec{\nabla} \\
(\mathcal{C P})\left(\rho_{e}\right)(\mathcal{C P})^{-1} & =-\rho_{e} \\
(\mathcal{C P})\left(\overrightarrow{j_{m}}\right)(\mathcal{C P})^{-1} & =-\overrightarrow{j_{m}} \\
(\mathcal{C P})\left(\phi_{e}\right)(\mathcal{C P})^{-1} & =-\phi_{e} \\
(\mathcal{C P})(\vec{D})(\mathcal{C P})^{-1} & =-\vec{D} \\
(\mathcal{C P})(\vec{B})(\mathcal{C P})^{-1} & =-\vec{B}
\end{aligned}
$$

Applying symmetry operation $\mathcal{C P}$ to equation (11), we see that the generalized Maxwell's equation in the homogeneous medium (11) are invariant under $\mathcal{C P}$ symmetry. Applying the $\mathcal{C P}$ symmetry operation to equations (4,7, like generalized field $(\vec{\psi})$, generalized scalar potential of dyon $(\phi)$, generalized vector potential of dyon $(\vec{V})$, generalized charge density of dyon $(\rho)$, generalized current density of dyon $(\vec{J})$ and generalized field current $(\vec{S})$ in homogeneous medium are changed as follows,

$$
\begin{aligned}
& (\mathcal{C P})(\vec{\psi})(\mathcal{C P})^{-1}=\vec{\psi} \\
& (\mathcal{C P})(\phi)(\mathcal{C P})^{-1}=-\phi ; \\
& (\mathcal{C P})(\vec{V})(\mathcal{C P})^{-1}=\vec{V} ; \\
& (\mathcal{C P})(\rho)(\mathcal{C P})^{-1}=-\rho ; \\
& (\mathcal{C P})(\vec{J})(\mathcal{C P})^{-1}=\vec{J} \text {; } \\
& (\mathcal{C P})(\vec{S})(\mathcal{C P})^{-1}=\vec{S}
\end{aligned}
$$

As such, the complex potential field equations (12) and the tensorial form of generalized Maxwell Dirac equation of dyons (13) in isotropic homogeneous medium are invariant under $\mathcal{C P}$ transformations. But the generalized field equation (16) and the Lorentz four - force equation of motion (18) of dyons in homogeneous isotropic medium are not invariant under $\mathcal{C P}$ symmetry.

\section{CPT and fields associated with dyons}

We have seen that there are three symmetries which usually, but not always, hold are those of charge conjugation $(\mathcal{C})$, parity $(\mathcal{P})$ and time reversal $(\mathcal{T})$. Examples in nature can be cited for the violation of each of these symmetries individually. It was thought for a time that $\mathcal{C P}$ (parity transformation plus charge conjugation) would always leave a system invariant, but the notable example of the neutral kaons has shown a slight violation of $\mathcal{C P}$ symmetry. We are left with the combination of all three, i.e. $\mathcal{C P} \mathcal{T}$, 
a profound symmetry which is assumed to be consistent with all known experimental observations. The velocity of the particle $(\vec{u})$, electric field $\overrightarrow{(E)}$ and the magnetic field $(\vec{B})$ are invariant under the combined operation of $\mathcal{C P} \mathcal{T}$,

$$
\begin{aligned}
& (\mathcal{C P} \mathcal{T})(\vec{u})(\mathcal{C P} T)^{-1}=\vec{u} \\
& (\mathcal{C P} \mathcal{T})(\vec{E})(\mathcal{C P} T)^{-1}=\vec{E} \\
& (\mathcal{C P} \mathcal{T})(\vec{B})(\mathcal{C P} T)^{-1}=\vec{B} .
\end{aligned}
$$

On the other hand, other physical variables are not invariant under the combined operation of $\mathcal{C P} \mathcal{T}$, i.e.

$$
\begin{aligned}
(\mathcal{C P} \mathcal{T})(x)(\mathcal{C P} T)^{-1} & =-x \\
(\mathcal{C P} \mathcal{T})(t)(\mathcal{C P} T)^{-1} & =-t \\
(\mathcal{C P} \mathcal{P})(e)(\mathcal{C P} T)^{-1} & =-e \\
(\mathcal{C P} \mathcal{T})(g)(\mathcal{C P} T)^{-1} & =-g \\
(\mathcal{C P} \mathcal{T})(\vec{\nabla})(\mathcal{C P} T)^{-1} & =-\vec{\nabla} \\
(\mathcal{C P} \mathcal{T})\left(\partial_{t}\right)(\mathcal{C P} T)^{-1} & =-\partial_{t} \\
(\mathcal{C P} \mathcal{T})\left(\rho_{e}\right)(\mathcal{C P} T)^{-1} & =-\rho_{e} \\
(\mathcal{C P} \mathcal{T})\left(\rho_{m}\right)(\mathcal{C P} T)^{-1} & =-\rho_{m} \\
(\mathcal{C P} \mathcal{T})\left(\overrightarrow{j_{e}}\right)(\mathcal{C P} T)^{-1} & =-\overrightarrow{j_{e}} \\
(\mathcal{C P} \mathcal{T})\left(\overrightarrow{j_{m}}\right)(\mathcal{C P} T)^{-1} & =-\overrightarrow{j_{m}} \\
(\mathcal{C P} \mathcal{T})\left(\phi_{e}\right)(\mathcal{C P} T)^{-1} & =-\phi_{e} \\
(\mathcal{C P} \mathcal{T})\left(\phi_{m}\right)(\mathcal{C P} T)^{-1} & =-\phi_{m} \\
(\mathcal{C P} \mathcal{T})(\vec{C})(\mathcal{C P} T)^{-1} & =-\vec{C} \\
(\mathcal{C P} \mathcal{T})(\vec{D})(\mathcal{C P} T)^{-1} & =-\vec{D}
\end{aligned}
$$

Consequently, if we apply the $\mathcal{C P} \mathcal{T}$ operation to equations (4, 7,9,10), we observe that the quantities like generalized field $(\vec{\psi})$ and generalized field current $(\vec{S})$ of dyons in homogeneous medium are invariant while generalized scalar potential of dyon $(\phi)$, generalized vector potential $(\vec{V})$, generalized charge density $(\rho)$ and generalized current density $(\vec{J})$ of dyon in homogeneous medium change are no more invariant i.e.

$$
\begin{array}{rlll}
(\mathcal{C P} \mathcal{T})(\vec{\psi})(\mathcal{C P} T)^{-1} & =\vec{\psi} ; \\
(\mathcal{C P} \mathcal{T})(\phi)(\mathcal{C P} T)^{-1} & = & -\phi ; \\
(\mathcal{C P} \mathcal{T})(\vec{V})(\mathcal{C P} T)^{-1} & = & -\vec{V} ; \\
(\mathcal{C P} \mathcal{T})(\rho)(\mathcal{C P} T)^{-1} & = & -\rho ; \\
(\mathcal{C P} \mathcal{T})(\vec{J})(\mathcal{C P} T)^{-1} & = & -\vec{J} ; \\
(\mathcal{C P} \mathcal{T})(\vec{S})(\mathcal{C P} T)^{-1} & \vec{S} .
\end{array}
$$


Accordingly, the complex potential field equations (12), the tensorial form of generalized Maxwell Dirac equations (13, 16) and the Lorentz four - force equation of motion (23) of dyon in homogeneous isotropic medium are invariant under the combined operation $(\mathcal{C P} \mathcal{T})$.

\section{Conclusion}

From the foregoing analysis, we have observed that the Maxwell's - Dirac equation (1) are invariant under discrete symmetries like parity $\mathcal{P}$, time reversal $\mathcal{T}$, charge conjugation $(\mathcal{C})$, parity - time reversal operation $\mathcal{P} \mathcal{T}$, charge conjugation - parity operation $\mathcal{C P}$ and combined operation $(\mathcal{C P} \mathcal{T})$. We have also seen that the complex nature of generalized field equation (16) and the Lorentz force equation of motion (18) of dyon in homogeneous medium loose their invariance under the discrete symmetries like parity $\mathcal{P}$, Time reversal $\mathcal{T}$, parity - time reversal operation $\mathcal{P} \mathcal{T}$ and charge conjugation - parity operation $\mathcal{C P}$. On the other hand, these equations are invariant under the combined operation $(\mathcal{C P} \mathcal{T})$. In other words, we may conclude that $\mathcal{C P} \mathcal{T}$ invariance is an exact symmetry for generalized fields of dyons and reproduces the consistent theory of electric charge (magnetic monopole) in the absence magnetic monopole (electric charge ) on dyons. As such the statement of Ludens - Pauli - Schwinger 25 that invariance under Lorentz transformations implies $\mathcal{C P} \mathcal{T}$ invariance holds good for the case of dyons in homogeneous isotropic medium.

ACKNOWLEDGMENT:- One of PSB is thankful to Chinese Academy of Sciences and Third World Academy of Sciences for awarding him CAS - TWAS Visiting Scholar Fellowship to pursue a research program in China.

\section{References}

[1] M. S. Sozzi, 'Discrete symmetries and CP violation', Oxford University Press (2008); Roy, A. , Resonance, $\underline{\mathbf{1 0}}$ (2005), 164.

[2] H. S. Leff and A. F. Rex, 'Maxwell's demon: entropy, information, computing', IOP publishing (1990) \& 'Maxwell's demon, 2: entropy, classical and quantum information', IOP publishing, (2003); Roger Penrose, 'The emperor's new mind: concerning computers, minds, and the laws of physics', Oxford University Press (2002).

[3] G. C. Branco, L. Lavoura and J. P. Silva, 'CP violation', Clarendon Press (1999); I. Bigi and A. Sanda, 'CP violation', Cambridge University Press (1999); Michael Beyer, 'CP Violation in Particle, Nuclear and Astrophysics', Springer (2002).

[4] R. F. Streater and A. S. Wightman, 'PCT, spin statistics and all that', Benjamin/Cummings (1964).

[5] E. P. Wigner, Z. Phys., $\underline{43}$ (1927), 624.

[6] J. D. Jackson, 'Classical Electrodynamics', New York, Willey (1975); J. Rosen, Am. J. Phys.,_41 (1973), 586. 
[7] N. F. Ramsey, Phys. Rev., 109 (1958), 225.

[8] L. Landau, Nuclear Phys., $\underline{\mathbf{3}}$ (1957), 127.

[9] T. D. Lee, 'Mimeographed lecture notes on conservation laws in Weak Interactions', Harward University, Physics Department (unpublished).

[10] J. Schwinger, Phys. Rev., $\underline{\mathbf{8 2}}$ (19510), 914.

[11] G Lüders, Kgl. Danske Videnskab. Selskab, Mat. fys. Medd., $\underline{\mathbf{2 8}}$ (1954).

[12] W. Pauli, 'Neils Bohr and the Development of Physics', McGraw- Hill Book Company, Inc. New York (1955).

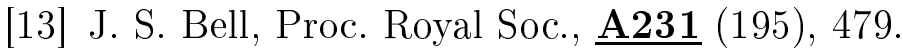

[14] Mark J. Hadely, 'A conserved parity operator', hep-ph/0406118v1.

[15] E. P. Shabalin, Physics - Uspekhi, $\underline{44}$ ( 2001), 895.

[16] Rashmi Gunawant and B. S. Rajput, Il Nuovo Cimento, $\underline{\mathbf{A} 86}$ (1985) 111.

[17] John W Norbury, Euro J. Phys., 11 (1990), 99.

[18] Jivan Singh, P. S. Bisht and O. P. S. Negi, Communication in Physics, 17 (2007), 83.

[19] Jivan Singh, P. S. Bisht and O. P. S. Negi, J. Phys. A: Math. And Theor., $\underline{40}$ (2007), 9137.

[20] Jivan Singh, P. S. Bisht and O. P. S. Negi, Communication in Physics, 17 (2007), 129.

[21] Jivan Singh, P. S. Bisht and O. P. S. Negi, J. Phys. A: Math. And Theor., $\underline{40}$ (2007), 11395.

[22] P. S. Bisht and O. P. S. Negi, Proceedings of 6th International conference on "Nuclear and Particle Physics, Egyptian Nuclear Physics Association, Luxor, Egypt from Nov. 17 - 21, 2007, Edited by M. N. H. Comsan (2008), 405 - 420.

[23] P. A. M. Dirac, Proc. Royal Society, $\underline{\mathbf{A 1 3 3}}$ (1931), 60.

[24] C. M. Bender and S. Boetter, Phys. Rev. Lett., 24 (1998), 5243; J. Phys. A: Math. Gen., $\underline{\mathbf{3 1}}$ (1998), L273.

[25] Georg Ludens, Wolfgang Pauli and Julian Schwinger http://hyperphysics.phy-astr.gsu.edu/HBASE/Particles/cpt.html 